\title{
Optimal Transmission with Per-antenna Power Constraints for Multiantenna Bidirectional Broadcast Channels
}

\author{
Phuong Le Cao, Tobias J. Oechtering and Mikael Skoglund
}

\author{
School of Electrical Engineering, KTH Royal Institute of Technology, Stockholm, Sweden
}

\begin{abstract}
This paper considers the optimal transmit strategy for multi-antenna bidirectional broadcast channels with perantenna power constraints. First, an equivalent formulation of the weighted rate sum maximization problem is provided. This allows us to come up with an effective solution to characterize the boundary of the capacity region which relies on the weighted rate sum optimal rate pair. To that end, an iterative algorithm to find the optimal transmit strategy is derived, the convergence to the optimum is proved, and a closed-form solution of the corresponding off-diagonal elements of the optimal transmit strategy is provided. Further, we provide a parametrization of the curved section of the capacity region. Finally, the theoretical results and algorithm performance are illustrated by numerical examples.
\end{abstract}

\section{INTRODUCTION}

Recently, relay networks have become more and more important in wireless communication systems since they efficiently extend their coverage range. It was shown in previous studies that bidirectional communication using the network coding idea can greatly improve the throughput in relaying [1], [2]. In bidirectional communication with decode-and-forward protocol, the whole transmission is divided into two phase: multiple-access phase (MAC) and broadcast phase (BC). Since the optimal transmit strategy for the MAC is well known [3], [4], we concentrate on bidirectional BC.

The optimal transmit strategies for the Gaussian MISO and MIMO bidirectional broadcast channels with sum power constraint have been well-studied [5], [6]. In [5], the authors show that, for the multiple-input single-output (MISO) bidirectional broadcast channel with sum power constraint, beamforming is always an optimal transmit strategy. It shows that an optimal transmit strategy for MISO bidirectional broadcast channel transmits into the subspace spanned by the channels only. In [6], the transmit covariance matrix optimization problem for the discrete memoryless multiple-input multiple-output (MIMO) Gaussian bidirectional broadcast channel is studied. A simple iterative fixed point algorithm is proposed to find the maximal weighted rate sum, which characterizes the capacity region of the bidirectional broadcast channel.

Practically, each antenna has its own power amplifier, which means the power allocation at the transmitter is usually done under the per-antenna power constraints instead of the sum power constraint. Indeed, optimal transmit strategies with average per-antenna power constraints have been investigated in both single-user [7]-[9] and multi-users [10]-[15] settings. In these works, the authors derived necessary and sufficient conditions for optimal MIMO transmission schemes and devel- oped iterative algorithms that converge to the optimal solution. However, the bidirectional broadcast channels with average per-antenna power constraints have not been considered yet.

In this paper, we study the optimal transmit strategy for a MISO bidirectional broadcast channel with per-antenna power constraints. We start by briefly introducing the system model of the MISO bidirectional broadcast channel and capacity region together with the power constraint. After that, we derive an equivalent problem formulation of the weighted rate sum maximization problem. Then, an iterative algorithm to compute the optimal transmit covariance matrix is provided. We also prove that the proposed algorithm converges to the global optimum. Lastly, we provide a parametrization of the curved section of the capacity region. ${ }^{1}$

\section{MISO BIDIRECTIONAL BROADCAST CHANNEL}

\section{A. System Model and Power Constraint}

Let $N_{t}$ be the number of transmit antennas at the relay node. The channel input-output relation between the relay node and node $i$ is given as follows:

$$
y_{i}=\mathbf{x}^{T} \mathbf{h}_{i}+z_{i}, \quad i=1,2
$$

where $\mathbf{x}=\left[x_{1}, \ldots, x_{N_{t}}\right]^{T} \in \mathbb{C}^{N_{t} \times 1}$ is the complex transmit signal vector, $\mathbf{h}_{i}=\left[h_{i 1}, \ldots, h_{i N_{t}}\right] \in \mathbb{C}^{N_{t} \times 1}, i=1,2$ are the channel coefficient vectors, and $z_{i}, i=1,2$ is the independent additive white complex Gaussian noise with power $\sigma^{2}$. The transmit covariance matrix of the Gaussian input $\mathbf{x}$ is defined as $\mathbf{Q}=\mathbb{E}\left[\mathbf{x} \mathbf{x}^{H}\right]$. In this paper, for simplicity, we assume that $\sigma^{2}=1$. Let $\mathcal{S}$ denote the set of all power allocations which satisfy the per-antenna power constraints, then $\mathcal{S}$ can be defined as $\mathcal{S}:=\left\{\mathbf{Q} \succeq 0: \mathbf{e}_{k}^{T} \mathbf{Q} \mathbf{e}_{k} \leq \hat{P}_{k}, k=1, \ldots, N_{t}\right\}$ where $\mathbf{e}_{k}$ is the $k^{t h}$ Cartesian unit vector.

\section{B. Capacity Region and Problem Statement}

In this part, we first introduce the capacity region of the Gaussian MISO bidirectional relay channel. After that, in perantenna power constraint domain, the problem to find the optimal transmit strategy is formulated.

Similarly to [5, Theorem 1], which considers an average sum power constraint, we obtain that the capacity region of

\footnotetext{
${ }^{1}$ Notation: We use bold lower-case letters for vectors, bold capital letters for matrices. The superscripts $(\cdot)^{T},(\cdot)^{*}$ and $(\cdot)^{H}$ stand for transpose, conjugate, and conjugate transpose. $\mathbb{R}, \mathbb{R}_{+}$and $\mathbb{C}$ are the sets of real, non-negative real and complex numbers. $\operatorname{dpch}(\cdot)$ denotes the downward positive comprehensive hull which is defined for the vector $\mathbf{x} \in \mathbb{R}_{+}^{2}$ by the $\operatorname{set} \operatorname{dpch}(\mathbf{x}):=\{\mathbf{y} \in$ $\left.\mathbb{R}_{+}^{2}: y_{i} \leq x_{i}, i=1,2\right\}$.
} 
the Gaussian MISO bidirectional broadcast channel, $\mathcal{C}_{B C}^{M I S O}$, with per-antenna power constraints is given by

$$
\mathcal{C}_{B C}^{M I S O}=\bigcup_{\mathbf{Q} \in \mathcal{S}} \operatorname{dpch}\left(\left[C_{1}(\mathbf{Q}), C_{2}(\mathbf{Q})\right]\right)
$$

with $C_{i}(\mathbf{Q}):=\log \left(1+\mathbf{h}_{i}^{H} \mathbf{Q} \mathbf{h}_{i}\right), \quad i=1,2$.

The boundary of the capacity region can be characterized by the set of weighted rate sum optimal rate pairs. The weighted rate sum for a given weight vector $\mathbf{w}=\left[w_{1}, w_{2}\right] \in \mathbb{R}_{+}^{2}$ with $w_{1}+w_{2}=1$, is given by

$$
R_{\sum}(\mathbf{Q}, \mathbf{w}):=w_{1} \log \left(1+\mathbf{h}_{1}^{H} \mathbf{Q} \mathbf{h}_{1}\right)+w_{2} \log \left(1+\mathbf{h}_{2}^{H} \mathbf{Q} \mathbf{h}_{2}\right) .
$$

The remaining question is to establish the optimal transmit strategy $\mathbf{Q}_{\text {opt }}(\mathbf{w})$ such that the weighted rate sum in (3) is maximized for a given vector $\mathbf{w}$. The optimization problem to find the maximal weighted rate sum for MISO bidirectional broadcast channels with per-antenna power constraint can be written as follows

$$
\max _{\mathbf{Q}} R_{\sum}(\mathbf{Q}, \mathbf{w}), \text { s.t. } \mathbf{Q} \in \mathcal{S}
$$

The boundary of the capacity region is given by the weighted rate sum optimal rate pairs where each rate pair is optimal for a certain weighted vector only. It is defined sectionwise and consists of two single-user optimal sections and the Pareto optimal section which is the curved part of the boundary similarly as in [5] for the sum power constraint problem. The tradeoff between two rates is controlled by optimal transmit strategy $\mathbf{Q}$. Regarding the property of the weight vector where $0 \leq w_{1}, w_{2} \leq 1$ and $w_{1}+w_{2}=1$, we know that when $\mathbf{w}=[1,0]$ and $\mathbf{w}=[0,1]$, the channel stays the same, but the optimization problem (3) becomes the single user problem.

The maximal unidirectional rate for user 1 is given by the optimization problem with $\mathbf{w}=[1,0]$, i.e., $R_{1, \text { opt }}^{(1)}:=$ $\max _{\mathbf{Q}} \log \left(1+\mathbf{h}_{1}^{H} \mathbf{Q} \mathbf{h}_{1}\right)$ subject to $\mathbf{Q} \in \mathcal{S}$. The rate pair $\left[R_{1, \text { opt }}^{(1)}, R_{2, \text { opt }}^{(1)}\right]$ can be found by solving this problem. The solution can be obtained from the single-user MISO channel with per-antenna power constraints in [7]. The optimal covariance matrix $\mathbf{Q}_{o p t}^{(1)}$ has rank one with $\mathbf{Q}_{o p t}^{(1)}=\sum_{k=1}^{N_{t}} \hat{P}_{k} \mathbf{v}_{1} \mathbf{v}_{1}^{H}$, and the beamforming vector $\mathbf{v}_{1}$ has the elements given as $v_{1 k}=\frac{\sqrt{\hat{P}_{k}}}{\sqrt{\sum_{k=1}^{N_{t}} \hat{P}_{k}}} \frac{h_{1 k}^{*}}{\left|h_{1 k}\right|}$. Therewith, the maximal unidirectional rate that user 1 can achieve is $R_{1, \text { opt }}^{(1)}=\log [1+$ $\left.\left(\sum_{k=1}^{N_{t}}\left|h_{1 k}\right| \sqrt{\hat{P}_{k}}\right)^{2}\right]$. Let $\rho_{k}:=v_{1 k} v_{2 k}^{*}=\frac{\hat{P}_{k}}{\sum_{k=1}^{N_{t}} \hat{P}_{k}} \frac{h_{1 k}^{*} h_{2 k}}{\left|h_{1 k}\right|\left|h_{2 k}\right|}$ denote the correlation between two elements $h_{1 k}$ and $h_{2 k}$ of channels $h_{1}$ and $h_{2}$. Then, the maximal rate user 2 can achieve is $R_{2, o p t}^{(1)}=\log \left[1+\left(\sum_{k=1}^{N_{t}}\left|\rho_{k}\right|\left|h_{2 k}\right| \sqrt{\hat{P}_{k}}\right)^{2}\right]$.

Similarly, for $\mathbf{w}=[0,1]$, the rate pair $\left[R_{1, o p t}^{(2)}, R_{2, o p t}^{(2)}\right]$ can be achieved with $\mathbf{Q}_{\text {opt }}^{(2)}=\sum_{k=1}^{N_{t}} \hat{P}_{k} \mathbf{v}_{2} \mathbf{v}_{2}^{H}$ where the beamforming vector $\mathbf{v}_{2}$ has the elements given as $v_{2 k}=$ $\frac{\sqrt{\hat{P}_{k}}}{\sqrt{\sum_{k=1}^{N_{t}} \hat{P}_{k}}} \frac{h_{2 k}^{*}}{\left|h_{2 k}\right|}$. The maximal unidirectional rate for user $2, R_{2, o p t}^{(2)}$, and the correponding $R_{1, o p t}^{(2)}$ are then given as $R_{2, o p t}^{(2)}=\log \left[1+\left(\sum_{k=1}^{N_{t}}\left|h_{2 k}\right| \sqrt{\hat{P}_{k}}\right)^{2}\right]$ and $R_{1, o p t}^{(2)}=\log [1+$ $\left.\left(\sum_{k=1}^{N_{t}}\left|\rho_{k}\right|\left|h_{1 k}\right| \sqrt{\hat{P}_{k}}\right)^{2}\right]$.
In the following, we provide the solution to find the optimal transmit strategy for weights $w_{1}, w_{2} \neq 0$. This also provides us a characterization of the capacity region.

\section{Optimal Transmit Strategy}

In this section, we derive an iterative algorithm solution to find the optimal transmit strategy of the problem (4) using an alternating optimization approach. To describe our approach, we reformulate our problem to a form that allows further analysis. To this end, we need the following lemma.

Lemma 1 ([16, Lemma 2, Scalar case]). Consider the function $f(D)=-D E+\log (D)+1$ where $D, E \in \mathbb{R}, E>0$. Then,

$$
\max _{D>0} f(D)=\log \left(E^{-1}\right) \text {, }
$$

with the optimum value $D^{*}=E^{-1}$.

By applying Lemma 1 to problem (4) with $E_{i}=1+\mathbf{h}_{i}^{H} \mathbf{Q} \mathbf{h}_{i}$, $i=1,2$, problem (4) can be expressed as

$$
\max _{\mathbf{Q} \in \mathcal{S}} \sum_{i=1}^{2} w_{i} \min _{D_{i}>0}\left(D_{i}\left(1+\mathbf{h}_{i}^{H} \mathbf{Q} \mathbf{h}_{i}\right)-\log \left(D_{i}\right)-1\right)
$$

That expression suggests the use of person-by-person optimality method for solving problem (6), i.e., at each iteration, the objective is maximized with respect to one variable while fixing the others. It is easy to see from (6) that by fixing either $\mathbf{Q}$ or $D_{i}, i=1,2$, the remaining problems are convex with respect to the other decision variable. Thus, we propose to solve the following optimization problems to obtain $\left(\mathbf{Q}_{o p t}^{[n]}, D_{i}^{[n]}\right)$ for $i=1,2$ in the $n^{t h}$ iteration.

$$
\begin{aligned}
D_{i}^{[n]} & =\arg \min _{D_{i}>0}\left(D_{i}\left(1+\mathbf{h}_{i}^{H} \mathbf{Q}^{[n-1]} \mathbf{h}_{i}\right)-\log \left(D_{i}\right)\right) \\
\mathbf{Q}_{\text {opt }}^{[n]} & =\arg \max _{\mathbf{Q} \in \mathcal{S}} \sum_{i=1}^{2} w_{i} D_{i}^{[n]} \mathbf{h}_{i}^{H} \mathbf{Q} \mathbf{h}_{i} .
\end{aligned}
$$

From Lemma 1, we know that, the closed-form solution of problem (7) can be computed as

$$
D_{i}^{[n]}=\left(1+\mathbf{h}_{i}^{H} \mathbf{Q}_{o p t}^{[n-1]} \mathbf{h}_{i}\right)^{-1} .
$$

As a basic property, for a given vector $\mathbf{w}$, the alternating optimization iterations yield a non-decreasing sequence of the weighted rate sums, i.e., $R_{\sum}\left(\mathbf{Q}_{o p t}^{[n]}, \mathbf{w}\right) \geq R_{\sum}\left(\mathbf{Q}_{o p t}^{[n-1]}, \mathbf{w}\right) \geq$ $\cdots \geq R_{\sum}\left(\mathbf{Q}_{o p t}^{[0]}, \mathbf{w}\right)$. An arbitrary initial point, which corresponding to $\mathbf{Q}_{o p t}^{[0]}$, is chosen such that the per-antenna power constraints are satisfied, i.e., $\mathbf{Q}_{o p t}^{[0]} \in \mathcal{S}$. Since the sequence of the weighted rate sums are bounded and monotonic increasing, convergence is guaranteed. Additionally, we can show that the sequence converges to the global optimum.

Proposition 1. Sequence $\left\{\boldsymbol{Q}_{\text {opt }}^{[n]}\right\}$ generated by the iterative algorithm given by (7)-(8) converges to the global optimum of problem (4).

Proof: The proof of Proposition 1 can be found in Appendix A. The key idea is to show that the limit is a stationary point of the convex optimization problem (4). 
In problem (8), the optimal $\mathbf{Q}_{o p t}^{[n]}$ must have the diagonal elements $q_{k k}^{[n]}=\hat{P}_{k}$. The problem remains to find the offdiagonal elements $q_{k l}^{[n]}, \forall k, l=1, \ldots, N_{t} ; k \neq l$.

Theorem 1. Let $\boldsymbol{Q}_{\text {opt }}^{[n]}$ be the optimal covariance matrix at the $n^{\text {th }}$ iteration. Then $\boldsymbol{Q}_{o p t}^{[n]}$ has the off-diagonal elements given as

$$
q_{k l}^{[n]}=\frac{\sum_{i=1}^{2} w_{i} D_{i}^{[n]} h_{i k}^{*} h_{i l}}{\left|\sum_{i=1}^{2} w_{i} D_{i}^{[n]} h_{i k}^{*} h_{i l}\right|} \sqrt{\hat{P}_{k} \hat{P}_{l}} .
$$

Proof: The proof of Theorem 1 can be found in Appendix B. The idea of the proof is that relaxing the semi-definite constraint to $2 \times 2$ principal minors, and show that the optimal covariance matrix of the relaxing problem is indeed the optimal covariance matrix of (8), similarly as in [7].

This leads to an iterative algorithm (IA) to find optimal transmit strategy for problem (4) (see Algorithm 1).

\section{Characterization OF CAPACITY REgION}

The most interesting part of the boundary is the curved section. This section corresponds to the set of Pareto optimal rate pairs which can be achieved by the following paremetrized optimal transmit strategies.

Theorem 2. The optimal transmit strategies $\boldsymbol{Q}_{\text {opt }}$ of the curved part can be parametrized as follows

$$
\boldsymbol{Q}_{\text {opt }}(t)=\arg \max _{\boldsymbol{Q} \in \mathcal{S}} t \boldsymbol{h}_{1}^{H} \boldsymbol{Q} \boldsymbol{h}_{1}+(1-t) \boldsymbol{h}_{2}^{H} \boldsymbol{Q} \boldsymbol{h}_{2},
$$

with $t \in[0,1]$.

C

Proof: The proof of Theorem 2 can be found in Appendix

Using the same argument in Theorem 1, we can identify the off-diagonal element of $Q_{o p t}(t)$ in (11) as

$$
q_{k l}(t)=\frac{t h_{1 k}^{*} h_{1 l}+(1-t) h_{2 k}^{*} h_{2 l}}{\left|t h_{1 k}^{*} h_{1 l}+(1-t) h_{2 k}^{*} h_{2 l}\right|} \sqrt{\hat{P}_{k} \hat{P}_{l}} .
$$

Remark 1. From the proof of Theorem 2, we see that $\boldsymbol{Q}_{\text {opt }}(t)$, $t \in[0,1]$, is an optimal transmit strategy for the weights

$$
w_{i}(t)=\frac{t\left(1+\boldsymbol{h}_{i}^{H} \boldsymbol{Q}_{o p t}(t) \boldsymbol{h}_{i}\right)}{t\left(1+\boldsymbol{h}_{i}^{H} \boldsymbol{Q}_{o p t}(t) \boldsymbol{h}_{i}\right)+(1-t)\left(1+\boldsymbol{h}_{j}^{H} \boldsymbol{Q}_{o p t}(t) \boldsymbol{h}_{j}\right)},
$$

$i, j=1,2, i \neq j$.

On the curved section of the boundary of capacity region, the rate pair $\mathbf{R}(t):=\left[R_{1}(t), R_{2}(t)\right]$ is achieved as $R_{i}(t):=\log \left(1+\mathbf{h}_{i}^{H} \mathbf{Q}(t) \mathbf{h}_{i}\right), i=1,2 . \mathbf{R}(1)$ and $\mathbf{R}(0)$ correspond to the two single-user optimal rate pairs $\left[R_{1, o p t}^{(1)}, R_{2, o p t}^{(1)}\right]$ and $\left[R_{1, o p t}^{(2)}, R_{2, o p t}^{(2)}\right]$, cf. Section II.B. Further on, $\mathbf{R}(t), t \in[0,1]$, parametrizes the curved section of the boundary of the capacity region and therewith all Pareto optimal rate pairs. Thus, following (2), the whole capacity region is characterized by the parametrization and given as $\mathcal{C}_{B C}^{M I S O}=\bigcup_{\mathbf{Q}(t) \in \mathcal{S}} \operatorname{dpch}\left(\left[R_{1}(t), R_{2}(t)\right]: R_{i}(t):=\log (1+\right.$ $\left.\left.\mathbf{h}_{i}^{H} \mathbf{Q}(t) \mathbf{h}_{i}\right), i=1,2, t \in[0,1]\right)$.

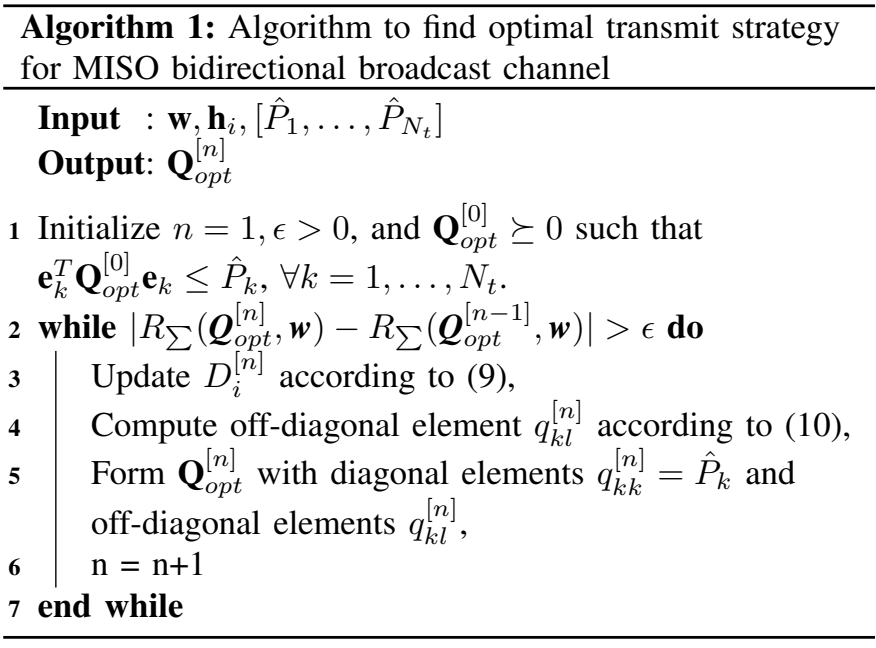

\section{NUMERICAL EXAMPLES}

In this section, we provide numerical examples to illustrate the theoretical results. We consider a MISO bidirectional broadcast channel with two antennas at the relay and one antenna each at the remaining nodes. The complex channel coefficients are denoted as $\mathbf{h}_{1}=[1.3,1.3 j]^{T}$ and $\mathbf{h}_{2}=$ $\left[1.2,-j e^{j \frac{\pi}{2}}\right]^{T}$. Two per-antenna powers are set as $\hat{P}_{1}=6$ and $\hat{P}_{2}=8$.

Fig. 1 depicts the capacity region for the MISO bidirectional broadcast channel with per-antenna power constraints. The solid line depicts the boundary of the capacity region. It is clear to see that the curved section corresponds to the weighted rate sum optimal rate pair. With a certain weight vector, we can obtain a rate pair. The point A and B in the Fig. 1 correspond to the case of $\mathbf{w}=[1,0]$ and $\mathbf{w}=[0,1]$ as denoted in section II.B, i.e., we have two corresponding rate pair $\left[R_{1, o p t}^{(1)}, R_{2, o p t}^{(1)}\right]=$ $[3.65,2.87]$ and $\left[R_{1, o p t}^{(2)}, R_{2, o p t}^{(2)}\right]=[3.02,3.53]$. The point $\mathrm{C}$ denotes the egalitarian solution, in this case the rate pair $\left[R_{1, \text { opt }}^{(3)}, R_{2, \text { opt }}^{(3)}\right]=[3.44,3.44]$ achieves at $\mathbf{w}=[0.4 .5,0.595]$.

In the next examples, we configure various number of antennas at the relay, and compare the working performance of our proposed algorithm and the standard CVX [17] (see http://cvxr.com/cvx/). The average running times of our algorithm (IA) and CVX in the same computer and same conditions $^{2}$, are shown in Table I. It can be seen that our proposed IA is much faster than CVX, particularly under a large number of transmit antennas.

\section{CONCLUSIONS}

In this paper, we studied the optimal transmit strategy for MISO bidirectional relay channel with average per-antenna power constraints. We derived an equivalent formulation of the optimization problem, and developed an iterative algorithm that converges to the global optimum. This also provided us a characterization of the capacity region. The capacity region, therefore, can be characterized. The efficiency of the

\footnotetext{
${ }^{2}$ The experiment is performed under: Processor - Intel Core i7-3740QM CPU @ 2.70GHz × 8; Memory - 8GB; OS: Ubuntu 14.04; Matlab R2015a.
} 


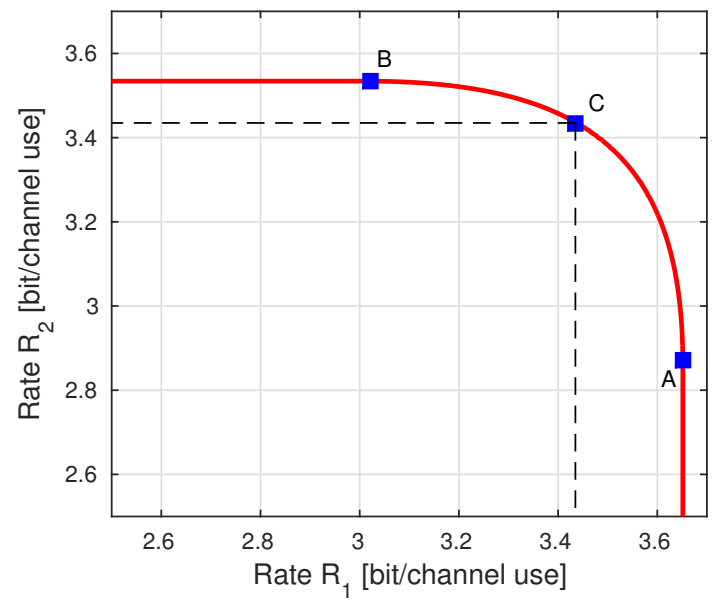

Fig. 1: Capacity region for MISO bidirectional broadcast channel with per-antenna power constraints $\hat{P}_{1}=6, \hat{P}_{2}=8$.

TABLE I: Average running time (in secs.) of iterative algorithm (IA) in comparison to $\mathrm{CVX}$

\begin{tabular}{|l|c|c|c|c|}
\hline \multirow{2}{*}{ Algorithm } & \multicolumn{4}{|c|}{ Number of antennas at the relay } \\
\cline { 2 - 5 } & 2 & 4 & 6 & 8 \\
\hline IA & 0.014 & 0.016 & 0.057 & 0.142 \\
\hline CVX & 1.425 & 1.899 & 3.243 & 4.827 \\
\hline
\end{tabular}

proposed approach has been demonstrated by a numerical example. Moreover, the performance analysis results show that the proposed algorithm performs much better than the standard CVX, in particular for the channel with a large number of transmit antennas.

\section{APPENDIX}

\section{A. Proof of Proposition 1}

Let $\phi_{1}(\mathbf{Q}, \mathbf{w}), \phi_{2}\left(\mathbf{Q}, D_{i}\right)$ and $\phi_{3}\left(\mathbf{Q}, \mathbf{w}, D_{i}\right)$, be the objective functions of (4), (7), and (8). Suppose that $\left(\mathbf{Q}^{\star}, D_{i}^{\star}\right), \forall i=1,2$, is a limit point of the sequence $\left\{\mathbf{Q}_{o p t}^{n}\right\}$ generated by (7)-(8). Because (7) is convex, following [17, Section 4.2 - pp.136145] we have

$$
\frac{\partial}{\partial D_{i}} \phi_{2}\left(\mathbf{Q}^{\star}, D_{i}^{\star}\right)\left(D_{i}-D_{i}^{\star}\right) \geq 0, \forall D_{i} \in \mathbb{R}, D_{i}>0 .
$$

This implies that for a given $\mathbf{Q}^{\star}$, the corresponding $D_{i}^{\star}, i=$ 1,2 , is uniquely given by $D_{i}^{\star}=\left(1+\mathbf{h}_{i}^{h} \mathbf{Q}^{\star} \mathbf{h}_{i}\right)^{-1}$. Thus,

$$
\begin{aligned}
& \frac{\partial \phi_{3}\left(\mathbf{Q}^{\star}, \mathbf{w}, D_{i}^{\star}\right)}{\partial \mathbf{Q}^{\star}}=\sum_{i=1}^{2} w_{i} D_{i}^{\star} \mathbf{h}_{i} \mathbf{h}_{i}^{H} \\
& =\sum_{i=1}^{2} w_{i} \mathbf{h}_{i}\left(1+\mathbf{h}_{i}^{h} \mathbf{Q}^{\star} \mathbf{h}_{i}\right)^{-1} \mathbf{h}_{i}^{H}=\frac{\partial \phi_{1}\left(\mathbf{Q}^{\star}, \mathbf{w}\right)}{\partial \mathbf{Q}^{\star}},
\end{aligned}
$$

i.e., $\mathbf{Q}^{\star}$ is a stationary point of problem (4). Since $\mathbf{Q}^{\star}$ satisfies also the sufficient Karush-Kuhn-Tucker (KKT) conditions of the convex optimization problem (4), it is also the global optimum.

\section{B. Proof of Theorem 1}

The main difficulty here is the positive semi-definite constraint $\mathbf{Q}^{[n]} \succeq 0$. This constraint is equivalent to having all principal minors of $\mathbf{Q}^{[n]}$ being positive semi-definite [18]. Let $\mathbf{M}^{[n]}$ be a relaxed version with semi-definite constraints involving only $2 \times 2$ principal minors of $\mathbf{Q}^{[n]}$ [7]. The minor matrix is obtained by removing $N_{t}-2$ columns, except $k$ and $l$, and the corresponding transposed $N_{t}-2$ rows of $\mathbf{Q}^{[n]}$. Then, $\mathbf{M}^{[n]}$ is given as $\mathbf{M}^{[n]}=\left[\begin{array}{ll}\hat{P}_{k} & q_{k l}^{[n] *} \\ q_{k l}^{[n]} & \hat{P}_{l}\end{array}\right], k, l=1, \ldots, N_{t}$, $k \neq l$. A relaxed version of the optimization problem (8) iteration is formed as

$$
\begin{gathered}
\max _{\mathbf{Q}} \sum_{i=1}^{2} w_{i} D_{i}^{[n]} \mathbf{h}_{i}^{H} \mathbf{Q} \mathbf{h}_{i}, \text { s.t. } q_{k k}^{[n]} \leq \hat{P}_{k}, k=1, \ldots, N_{t} \\
\mathbf{M}^{[n]} \succeq 0 .
\end{gathered}
$$

It is clear to see that if the optimal $\mathbf{Q}_{o p t}^{[n]}$ of this relaxed problem is positive semi-definite, then it is also the optimal solution of (8) [7]. The Lagrangian for problem (16) is given by

$$
\begin{aligned}
\mathcal{L} & =\sum_{i=1}^{2} w_{i} D_{i}^{[n]} \mathbf{h}_{i}^{H} \mathbf{Q} \mathbf{h}_{i} \\
& -\sum_{k \neq l} \lambda_{k l}\left(\left|q_{k l}^{[n]}\right|^{2}-\hat{P}_{k} \hat{P}_{l}\right)-\sum_{k} \mu_{k}\left(q_{k k}^{[n]}-\hat{P}_{k}\right),
\end{aligned}
$$

where $\lambda_{k l}$ and $\mu_{k}$ are the Lagrange multipliers at $n_{t h}$ iteration, and $k, l=1, \ldots, N_{t}$. Taking the first derivative of (17) and set it equal to zero, we have

$$
\frac{\partial \mathcal{L}}{\partial q_{k l}^{[n]}}=\sum_{i=1}^{2} w_{i} D_{i}^{[n]} h_{i k}^{*} h_{i l}-\lambda_{k l} q_{k l}^{[n]} \stackrel{!}{=} 0
$$

or equivalently

$$
q_{k l}^{[n]}=\frac{\sum_{i=1}^{2} w_{i} D_{i}^{[n]} h_{i k}^{*} h_{i l}}{\lambda_{k l}} .
$$

The optimal value of $q_{k l}^{[n]}$ is obtained when its constraint is satisfied with equality, i.e., $\left|q_{k l}^{[n]}\right|^{2}=\hat{P}_{k} \hat{P}_{l}$. By combining this condition with (19), we have the value of $q_{k l}^{[n]}$ as in (10). Since $\lambda_{k l}>0$ then second derivative $\frac{\partial^{2} \mathcal{L}}{\partial\left(q_{k l}^{[n]}\right)^{2}}=-\lambda_{k l}<0$. This implies that $\mathbf{Q}_{o p t}^{[n]}$ with $q_{k l}^{[n]}$ above is the maximum point of (16). Besides that, since the optimal $\mathbf{Q}_{o p t}^{[n]}$ is positive semidefinite, it is also the optimal solution of (8).

\section{Proof of Theorem 2}

The proof can be divided in two following steps:

Step 1: We show that there exists a $t \in[0,1]$ such that $\mathbf{Q}_{\text {opt }}(t)=\mathbf{Q}^{\star}$ for a given $\left(\mathbf{w}, D_{i}^{\star}\right)$. Suppose that for a given weight $\mathbf{w}=\left[w_{1}, w_{2}\right],\left(\mathbf{Q}^{\star}, D_{i}^{\star}\right), \forall i=1,2$, is the optimal solution of (8). Then, by letting $t=\frac{w_{1} D_{1}^{\star}}{\sum_{i=1}^{2} w_{i} D_{i}^{\star}}, t \in[0,1]$, we have $\mathbf{Q}_{\text {opt }}(t)=\arg \max _{\mathbf{Q} \in \mathcal{S}} t \mathbf{h}_{1}^{H} \mathbf{Q} \mathbf{h}_{1}+(1-t) \mathbf{h}_{2}^{H} \mathbf{Q} \mathbf{h}_{2}=\mathbf{Q}^{\star}$.

Step 2: We show that there exists a weight vector $\mathbf{w}=$ $\left[w_{1}, w_{2}\right], w_{1}+w_{2}=1$ such that the optimal $\mathbf{Q}^{\star}(\mathbf{w})=\mathbf{Q}_{o p t}(t)$ for $t \in[0,1]$. We know from (9) that, at the optimum, $D_{i}^{\star}(t)$ is given as $D_{i}^{\star}(t)=\left(1+\mathbf{h}_{i}^{H} \mathbf{Q}_{\text {opt }}(t) \mathbf{h}_{i}\right)^{-1}, \forall i=1,2$. Then, by letting $w_{i}(t)=\frac{t D_{j}^{\star}(t)}{(1-t) D_{i}^{\star}(t)+t D_{j}^{\star}(t)}, \forall i, j=1,2, i \neq j$, we have $\mathbf{Q}^{\star}(\mathbf{w}(t))=\arg \max _{\mathbf{Q} \in \mathcal{S}} \sum_{i=1}^{2} w_{i}(t) D_{i}^{\star}(t) \mathbf{h}_{i}^{H} \mathbf{Q} \mathbf{h}_{i}=\mathbf{Q}_{o p t}(t)$. 


\section{REFERENCES}

[1] P. Larsson, N. Johansson, and K.-E. Sunell, "Coded Bi-directional Relaying," in 5th Scandinavian Workshop on Wireless Ad-hoc Networks (AdHoc'05), Stockholm, Sweden, May 2005.

[2] Y. Wu, P. A. Chou, and S. Y. Kung, "Information exchange in wireless networks with network coding and physical-layer broadcast," in 39th Conf. Inf. Sci. Syst., Baltimore, Maryland, March 2005.

[3] A. Goldsmith, S. A. Jafar, N. Jindal, and S. Vishwanath, "Capacity limits of MIMO channels," IEEE Journal on Selected Areas in Communications, 2003.

[4] E. Biglieri, R. Calderbank, A. Constantinides, A. Goldsmith, A. Paulraj, and H. V. Poor, MIMO Wireless Communications. Cambridge University Press, 2007.

[5] T. J. Oechtering, R. F. Wyrembelski, and H. Boche, "Multiantenna bidirectional broadcast channels - Optimal transmit strategies," IEEE Trans. on Signal Process., vol. 57, no. 5, pp. 1948-1958, May 2009.

[6] T. J. Oechtering, E. A. Jorswieck, R. F. Wyrembelski, and H. Boche, "On the Optimal Transmit Strategy for the MIMO Bidirectional Broadcast Channel," IEEE Trans. on Communications, vol. 57, no. 12, pp. $3817-$ 3826, Dec.

[7] M. Vu, "MISO capacity with per-antenna power constraint," IEEE Trans. on Communications, vol. 59, no. 5, pp. 1268-1274, May 2011.

[8] — "MIMO Capacity with Per-antenna power constraint," in Global Communications Conference (GLOBECOM), 2011.

[9] Z. Pi, "Optimal MIMO transmission with per-antenna power constraint," in Global Communications Conference (GLOBECOM), Dec 2012.

[10] W. Yu and T. Lan, "Transmitter optimization for the multi-antenna downlink with per-antenna power constraint," IEEE Trans. on Signal Process., vol. 55, no. 6, pp. 2646-2660, June 2007.

[11] S. Shi, M. Schubert, and H. Boche, "Per-antenna power constrained rate optimization for multiuser MIMO systems," in Proc. International ITG Workshop Smart Antennas., 2008.

[12] K. Karakayali, R. Yates, G. Foschini, and R. Valenzuela, "Optimum zero-forcing beamforming with per-antenna power constraint," in Proc. of International Symposium on Information Theory (ISIT), June 2007.

[13] F. Boccardi and H. Huang, "Optimum power allocation for the MIMOBC Zero-forcing precoder with Per-antenna power constraints," in Proc. Conf, Inf. Science System (CISS), Mar 2006.

[14] R. Zhang, "Cooperative multi-cell block diagonalization per-base-station power constraints," IEEE Journal on Selected Areas in Communications, vol. 28, no. 9, pp. 1435-1445, Dec 2010.

[15] A. Tölli, M. Codreanu, and M. Juntti, "Linear multiuser MIMO transceiver design with quality of service and per-antenna power constraints," IEEE Trans. on Signal Process., vol. 56, no. 7, pp. 3049-3055, Jul 2008.

[16] J. Jose, N. Prasad, M. Khojastepour, and S. Rangarajan, "On robust weighted-sum rate maximixation in MIMO interference networks," in IEEE International Conference on Communications (ICC), 2011.

[17] S. Boyd and L. Vandenberghe, Convex Optimization. Cambridge University Press, 2009.

[18] J. Prussing, "The principal minor test for semidefinite matrices," Journal of Guidance, Control and Dynamics, vol. 9, no. 1, pp. 121-122, Jan-Feb 1986. 